\title{
Theoretical Calculations of Cohesive and Electronic Properties of Quaternary AlGaInN Alloys
}

\author{
M. ŁopUszYŃSKI ${ }^{a}$, J. BARTOSZEK $^{b}$ AND J.A. MAJEWSKI $^{b}$ \\ ${ }^{a}$ Interdisciplinary Centre for Mathematical and Computational Modeling \\ Warsaw University, Pawińskiego 5A, 02-106 Warsaw, Poland \\ ${ }^{b}$ Institute of Theoretical Physics, Faculty of Physics, Warsaw University \\ Hoża 69, 00-681 Warszawa, Poland
}

\begin{abstract}
We present theoretical studies of the AlGaInN nitride quaternary alloys. The studies are based on ab initio calculations performed within the density functional theory and virtual crystal approximation. The equilibrium lattice constants, bulk moduli, and elastic constants were calculated for the whole possible range of concentrations of the alloy constituents. The theoretical values were then fitted with second- and third-order polynomials. For all properties studied, the considerable bowing was observed.
\end{abstract}

PACS numbers: 61.66.Dk, 61.66.-f, 62.20.Dc, 64.30.+t

\section{Introduction}

AlGaInN quaternary alloys have become important components of the optoelectronic devices such as light emitting and laser diodes (LEDs and LDs) in green, blue, and UV regime [1-3]. The main idea of employing AlGaInN alloys lies in the possibility of tuning the band gap of the alloy in the range from green to UV light emission with a suitable In and $\mathrm{Al}$ concentration, simultaneously keeping the alloy lattice matched to the GaN substrate. However, the progress in development of devices based on these alloys is hampered by the very poor knowledge of the cohesive and electronic properties of these technologically important alloys. This concerns also the reliable modeling of functional systems employing quaternary AlGaInN alloys.

To close this gap, in the present paper we have undertaken complex theoretical studies of the electronic and cohesive properties of the quaternary AlGaInN alloys employing virtual crystal approximation (VCA) [4]. Our studies are based on $a b$ initio calculations in the framework of the density functional theory (DFT) 
within local density approximation (LDA) to the exchange and correlation functional and pseudopotentials to describe electron-ion interaction. This approach turned out to be very successful in describing cohesive and electronic properties of the constituent bulks, AlN, GaN, and InN. The LDA + VCA computational scheme has also led to valuable predictions of the cohesive properties of many alloys [5]. Here, we provide the theoretical predictions of the cohesive properties of the cubic quaternary $\mathrm{Al}_{x} \mathrm{Ga}_{y} \mathrm{In}_{1-x-y} \mathrm{~N}$ alloys, such as the equilibrium lattice constants, bulk moduli, and elastic constants, for the whole possible range of the In and $\mathrm{Al}$ concentration, $x$ and $y$, respectively.

The technologically important nitride alloys are usually grown in the wurtzite crystallographic structure. However, the ab initio calculations for the wurtzite structure are much more complex than for the simple cubic one. In such situation, there is a common practice to employ formulae that allow a reliable estimation of elastic constants for the wurtzite structure from the calculations of the cubic systems $[6,7]$. Therefore, the theoretically predicted cohesive properties for cubic quaternary alloys could close the gap of lacking knowledge and provide valuable data for modeling nitride devices based on quaternary alloys. The paper is organized as follows. In Sect. 2, we describe very shortly the computational procedure, in Sect. 3 we discuss the obtained results, and finally we conclude the paper in Sect. 4.

\section{Computational details}

The ab initio calculations of cohesive properties of quaternary alloys were performed employing DFT with LDA [8] for exchange and correlation with parameterization of Perdew and Zunger [9]. The electron-ion interaction has been described employing separable Kleinman-Bylander pseudopotentials [10, 11]. This scheme allows for an easy implementation of the VCA, and it leads to only minor modifications for standard plane-waves based numerical codes. For a given atom, the nonlocal separable pseudopotential reads

$$
\hat{V}_{\mathrm{ion}}^{\mathrm{ps}}=\hat{V}_{\mathrm{loc}}+\sum_{l j m} \frac{\left|f_{l j m}\right\rangle\left\langle f_{l j m}\right|}{W_{l j}},
$$

where the so-called projector $\left|f_{l j m}\right\rangle=\left|\delta V_{l j} R_{l j} Y_{l j m}\right\rangle, \delta V_{l j}=V_{l j}^{\mathrm{ps}}-V_{\mathrm{loc}}$, and normalization factors $W_{l j}=\left\langle R_{l j}\left|\delta V_{l j}\right| R_{l j}\right\rangle$, whereas $R_{l j}$ and $V_{l j}^{\text {ps }}$ fulfill the pseudopotential equation

$$
\left(-\frac{\mathrm{d}^{2}}{\mathrm{~d} r^{2}}+\frac{l(l+1)}{r^{2}}+V_{l j}^{\mathrm{ps}}(r)-\varepsilon_{l j}\right) R_{l j}(r)=0,
$$

with $l, j, m$ being angular, total, and magnetic angular momentum quantum numbers, respectively, $\varepsilon_{l j}$ is one particle atomic energy of state $(l, j)$, and the local part of the pseudopotential, $V_{\text {loc }}$, is usually taken as one of the pseudopotential components $V_{l j}^{\mathrm{ps}}$. In the AlGaInN quaternary alloys studied in this paper, each site of the cation sublattice is occupied by one of three cations $\mathrm{In}, \mathrm{Al}$, or Ga. In 
the spirit of the VCA, the effective pseudopotential of the cationic site is taken as a mixture of the pseudopotentials of the individual cations $\alpha$ weighted by their corresponding concentrations $x_{\alpha}$ :

$$
\hat{V}_{\mathrm{VCA}}^{\text {cation }}=\sum_{\alpha} x_{k} a \hat{V}_{\mathrm{loc}}^{\alpha}+\sum_{\alpha, I} x_{\alpha} \frac{\left|f_{I}^{\alpha}\right\rangle\left\langle f_{I}^{\alpha}\right|}{W_{I}},
$$

where indices $l, j, m$ have been lumped into one index $I$. This form of the VCA pseudopotential allows for a very easy calculation of the matrix elements in the plane-wave basis. The computational scheme for separable pseudopotentials employed in this paper is much simpler than the method proposed recently [12].

\section{Results}

The predicted equilibrium lattice constants and bulk moduli of the cubic quaternary AlGaInN alloys are depicted in Figs. 1 and 2, respectively. The devi-

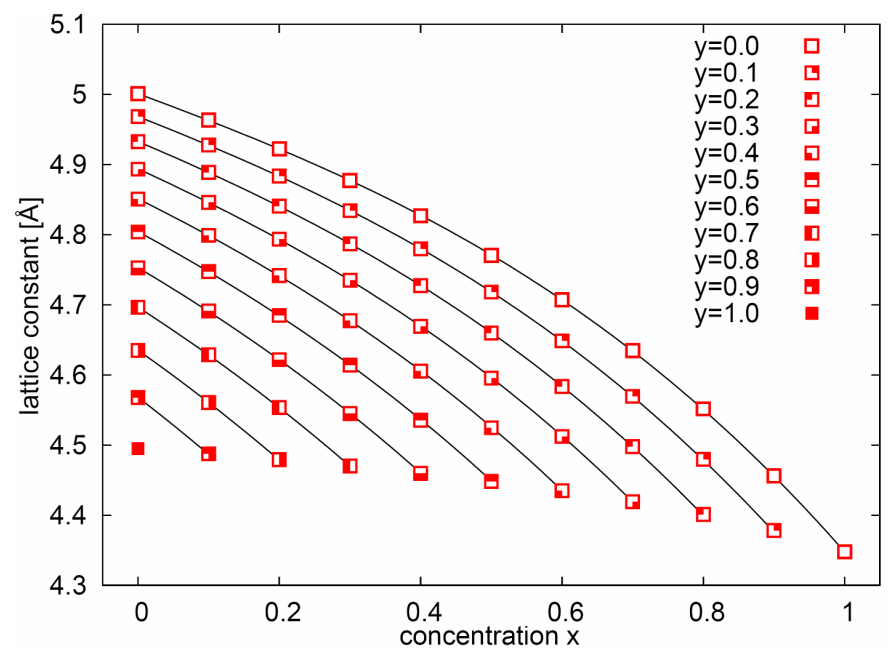

Fig. 1. The predicted equilibrium lattice constants for cubic $\mathrm{Al}_{x} \mathrm{Ga}_{y} \mathrm{In}_{1-y-x} \mathrm{~N}$ quaternary alloys as a function of $\mathrm{Al}$ concentration $x$ for a series of Ga concentrations $y$ (various squares) from $y=0$ to $y=1.0$ with step 0.1 . Let us note that only such values of $x$ are meaningful that lead to the positive concentrations for In cations. The solid lines represent third-order polynomial fit to the numerically computed values (see the text).

ation from the linear concentration dependence (Vegard-like law) and considerable bowing are apparent. For a given physical quantity $W$ that characterizes quaternary alloy, we define the bowing function $B(x, y)$ as the deviation from the linear terms

$$
W(x, y)=x W(\mathrm{AlN})+y W(\mathrm{GaN})+(1-x-y) W(\operatorname{InN})+B(x, y) .
$$

We have used two models of the bowing term; one containing second-order terms 


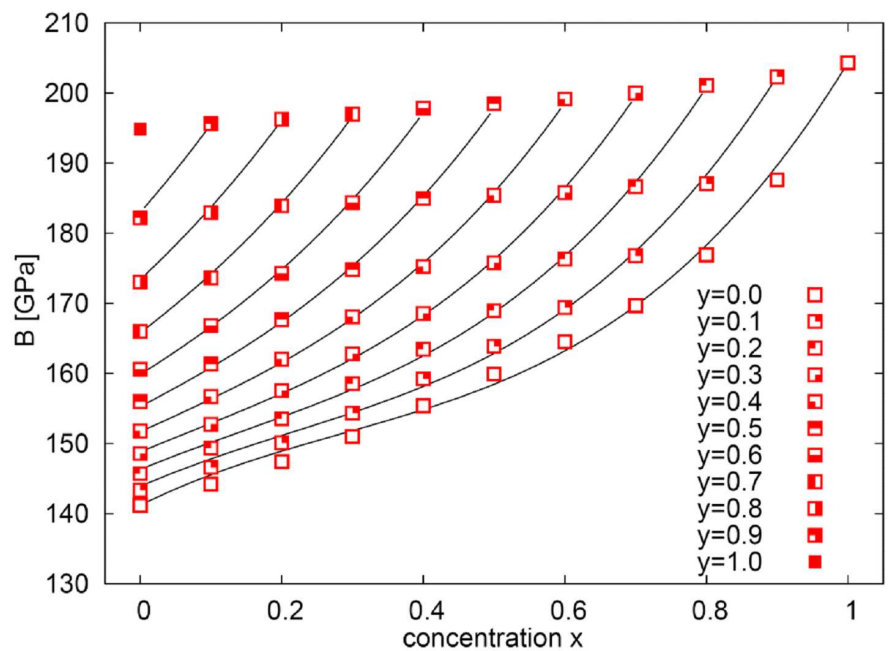

Fig. 2. The predicted bulk moduli for cubic $\mathrm{Al}_{x} \mathrm{Ga}_{y} \operatorname{In}_{1-y-x} \mathrm{~N}$ quaternary alloys as a function of $\mathrm{Al}$ concentration $x$ for a series of Ga concentrations $y$ between 0.0 and 1.0. The validity range of variable $x$ and meaning of solid lines were explained in caption to Fig. 1.

only, $B^{(1)}(x, y)$, and the second one with third-order terms, $B^{(2)}(x, y)$,

$$
B^{(1)}(x, y)=A_{0}^{(1)} x(1-x)+A_{1}^{(1)} y(1-y)+B_{10}^{(1)} x y
$$

and

$$
\begin{aligned}
& B^{(2)}(x, y)=A_{0}^{(2)} x(1-x)+A_{1}^{(2)} y(1-y)+B_{10}^{(2)} x y \\
& \quad+B_{00} x^{2}(1-x)+B_{11} y^{2}(1-y)+C_{12} x y^{2}+C_{21} x^{2} y
\end{aligned}
$$

where constants $A_{0}^{(1)}, A_{1}^{(1)}, B_{10}^{(1)}, A_{0}^{(2)}, A_{1}^{(2)}, B_{10}^{(2)}, B_{00}, B_{11}, C_{12}$, and $C_{21}$ have been determined by fitting to the values from ab initio calculations. The application of the Vegard-like law for lattice constants and bulk moduli of alloys leads to maximum errors of $2.1 \%$ and $9 \%$, respectively. The quality of the fits improves considerably, if one uses bowing terms, being equal to $0.24 \%$ and $3 \%$ for $B^{(1)}(x, y)$ model and $0.05 \%$ and $1 \%$ for $B^{(2)}(x, y)$ term. The calculated values of the constants describing the bowing terms in Eq. (4) are as follows: for the lattice constant (in $\AA$ ) $A_{0}^{(1)}=0.39, A_{1}^{(1)}=0.21, B_{10}^{(1)}=-0.48, A_{0}^{(2)}=0.28$, $A_{1}^{(2)}=0.19, B_{10}^{(2)}=-0.23, B_{00}=0.21, B_{11}=0.06, C_{12}=-0.25, C_{21}=-0.30$, and for the bulk modulus (in GPa) $A_{0}^{(1)}=-57.89, A_{1}^{(1)}=-49.68, B_{10}^{(1)}=93.09$, $A_{0}^{(2)}=-12.86, A_{1}^{(2)}=-24.44, B_{10}^{(2)}=-77.95, B_{00}=-88.63, B_{11}=-53.19$, $C_{12}=166.79, C_{21}=191.39$.

We have also calculated elastic constants for the $\mathrm{Al}_{x} \mathrm{Ga}_{y} \mathrm{In}_{1-y-x} \mathrm{~N}$ quaternary alloys. For each pair of concentrations $(x, y)$ we deformed infinitesimally cubic unit cell with equilibrium lattice constant of the alloy $a(x, y)$ and calculated the total energy of the deformed lattice. The difference in the total energies of 


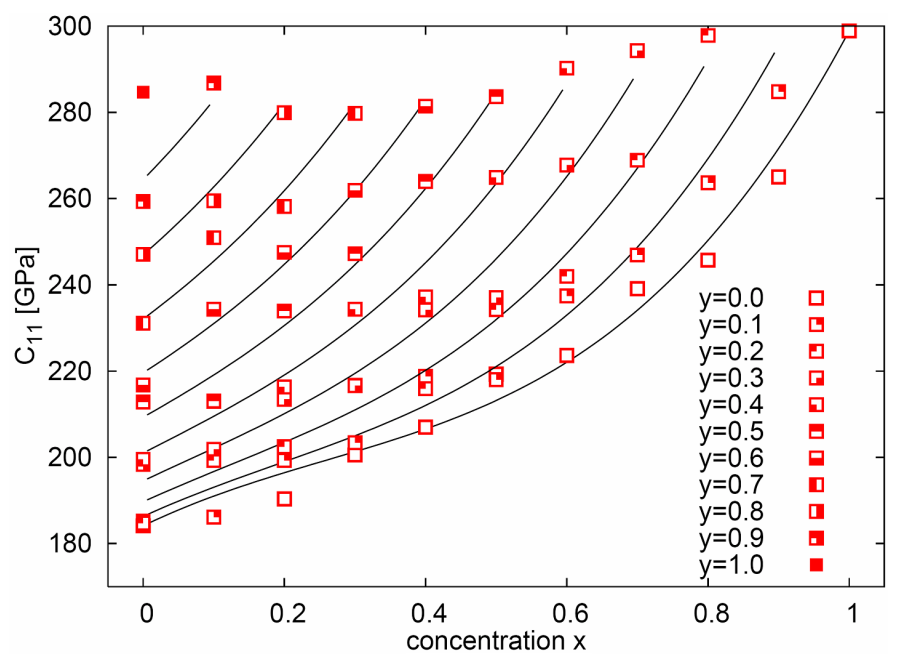

Fig. 3. The elastic constant $C_{11}$ for $\mathrm{Al}_{x} \operatorname{In}_{y} \mathrm{Ga}_{1-y-x} \mathrm{~N}$ quaternary alloy as a function of Al concentration $x$ for a series of In concentrations $y$ in the range from 0.0 to 1.0. The solid lines represent the third-order polynomial fit to the theoretical values.

the deformed and not deformed lattices was then fitted to the corresponding expressions from the elasticity theory [7]. In this manner we have obtained elastic constants $C_{11}(x, y), C_{12}(x, y)$, and $C_{44}(x, y)$ for the whole range of the possible concentrations of the alloy constituents. In Fig. 3 , the calculated elastic constants $C_{11}(x, y)$ are depicted together with the third-order polynomial fit of this quantity. One can see that the quality of the fit is not as good as in the case of lattice constant or bulk modulus and the error exceeds $12 \%$. Just to illustrate the range of possible values of the elastic constants in the cubic quaternary AlGaInN alloy, we present the so-called ternary plots for $C_{11}(x, y)$ and $C_{12}(x, y)$ elastic constants in Fig. 4. We would like to stress that all calculated quantities for binary alloys, i.e., AlN, GaN, and InN, agree very well with values from previous theoretical DFT calculations and with experimental values $[7,13]$.

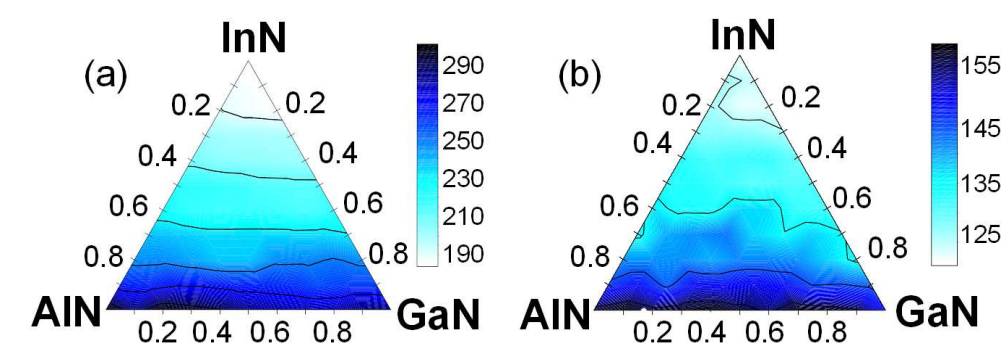

Fig. 4. Ternary plots for elastic constant $C_{11}$ (a) and $C_{12}$ (b) (in GPa) for AlGaInN quaternary alloys. 


\section{Conclusions}

The $a b$ initio calculations in the framework of LDA and VCA provide theoretical predictions for equilibrium lattice constants and elastic constants of the quaternary AlGaInN alloys. These quantities exhibit pronounced bowing. The calculated values constitute a solid basis for modeling of functional devices utilizing the nitride quaternary alloys.

\section{Acknowledgments}

This work was supported by the Polish Ministry of Science and Higher Education (grant No. 1P03B 037 29).

\section{References}

[1] M.E. Aumer, S.F. LeBoeuf, S.M. Bedair, M. Smith, J.Y. Lin, H.X. Jiang, Appl. Phys. Lett. 77, 821 (2000).

[2] A. Asan, R. McClintock, K. Mayes, S.R. Darvish, P. Kung, M. Razeghi, Appl. Phys. Lett. 81, 801 (2002).

[3] H. Hirayama, K. Akita, T. Kyono, T. Nakamura, Phys. Status Solidi A 201, 2639 (2004).

[4] L. Nørdheim, Ann. Phys. (Leipzig) 9, 607 (1931); T. Muto, Sci. Pap. Inst. Phys. Chem. Res. (Jpn) 34, 377 (1938).

[5] L. Belliche, D. Vanderbilt, Phys. Rev. B 61, 7877 (2000); Rh. Bouamama, K. Daoud, K. Kassaki, Model. Simul. Mater. Sci. Eng. 13, 1153 (2005).

[6] R.M. Martin, Phys. Rev. B 6, 4546 (1972).

[7] S.P. Łepkowski, J.A. Majewski, G. Jurczak, Phys. Rev. B 72, 245201 (2005).

[8] W. Kohn, I.J. Sham, Phys. Rev. A 140, 1133 (1965).

[9] J.P. Perdew, A. Zunger, Phys. Rev. B 23, 5048 (1981).

[10] B.M. Bylander, L. Kleinman, Phys. Rev. B 41, 7868 (1990).

[11] J.A. Majewski, S. Birner, A. Trellakis, M. Sabathil, P. Vogl, Phys. Status Solidi C 1, 2003 (2004).

[12] N.J. Ramer, A.M. Rappe, arXiv:cond-mat/9909032v3.

[13] M. Łopuszyński, J.A. Majewski, Phys. Rev. B 76, 045202 (2007). 